Preface

\title{
Quality in Hemostasis and Thrombosis, Part II
}

\author{
Giuseppe Lippi, MD ${ }^{1}$ Mario Plebani, MD ${ }^{2}$ Emmanuel J. Favaloro, PhD, FFSc (RCPA) ${ }^{3}$ \\ 1 U.O. Diagnostica Ematochimica, Azienda Ospedaliero-Universitaria \\ di Parma, Parma, Italy \\ 2 Dipartimento Medicina di Laboratorio, Azienda Ospedaliera- \\ Università di Padova, Padova, Italy \\ ${ }^{3}$ Department of Haematology, Institute of Clinical Pathology and \\ Medical Research (ICPMR), Westmead Hospital, Westmead, Australia
}

Semin Thromb Hemost 2013;39:229-232.

Welcome to the latest issue of Seminars in Thrombosis \& Hemostasis, the second to be devoted to the concept of "quality" within the field of thrombosis and hemostasis. ${ }^{1}$ This series of issues is intended to thoughtfully cover several clinical and laboratory issues related to diagnosis, management, and specific testing in the field of hemostasis.

The concept of quality in health care is often misinterpreted. According to the US Institute of Medicine, it should be intended as the extent to which health care services are capable of providing the best outcomes. ${ }^{2}$ Pursuing total quality in health care should therefore be seen as an attitude, an inclination that permeates an entire organization. The setting of thrombosis and hemostasis is not an exception to this rule, inasmuch as clinical, diagnostic, and therapeutic efforts should be directed toward the best possible outcome in patients with either bleeding or thrombotic disorders.

The first contribution of this issue is by the Guest Editors, ${ }^{3}$ and it covers the regulation of diagnostic products, termed "in vitro diagnostic devices" or IVDs by the regulators. The diagnostic or test-performance process, as associated to the evaluation or diagnosis of hemostasis and thrombosis and related disorders, is influenced or controlled by the activity of a large number of professional, expert, and government organizations. These may be involved in driving standardization or harmonization, providing professional or expert guidelines, or in regulation of therapeutic products. Although all organizations involved in this activity would propose an intention to drive improvements in diagnostics and human health, and although there are benefits to the overall process of standardization and regulation for tests of hemostasis and thrombosis, it should also be recognized that there are several specific problems and limitations to this process. As highlighted in this report, which specifically relates to the regulation of IVDs, several case studies are used as examples to show that regulation, aimed to reduce risks associated with the implementation of diagnostic testing, may instead en- courage the adverse outcomes of locking out clinically useful, new, and improved technologies, and locking in old and outdated technologies. This has potential for significant adverse outcomes related to the clinical diagnosis and management of hemostasis- and thrombosis-related disorders. This article represents an extension and update of previous reports from these authors. ${ }^{4-7}$ Another related article (Part 2) by these authors, and devoted to regulation of therapeutic agents in the field of thrombosis and hemostasis, is planned for a future issue of Seminars in Thrombosis \& Hemostasis.

Arterial and venous thromboses represent the most prevalent disorders worldwide, the former ranked first among the causes of death and disability in western countries ${ }^{8}$ and the latter being the most frequent complication of prolonged hospitalization. ${ }^{9}$ The second article of this quality issue, by Simoens and Huys, ${ }^{10}$ is therefore devoted to an intriguing, clinically significant and "quality" topic related to the therapy of thrombotic disorders, and in part, also related to regulatory considerations of drug compounds, that is, the market entry of biosimilar low-molecular-weight heparin (LMWH) formulations in Europe. The article contains a comprehensive analysis of regulatory requirements, pricing, reimbursement, prescribing, and dispensing of LMWHs. Among the leading issues currently driving the market, the authors identified some regulatory requirements such as quality dossier, biological and clinical investigations, comparability exercises, along with costs, prices, and the adverse impact of the heparin contamination crisis. Even more interestingly, the authors concluded that demand-side incentives for biosimilar LMWHs are largely lacking, whereas several issues persist about the interchangeability and substitution between originator and biosimilar LMWHs, all of which impact the potential differential "quality" of LMWH therapy, depending on the compounds used.

The extra-analytical phases of testing (i.e., all those "preanalytical" activities that anticipates testing and those
Address for correspondence

Giuseppe Lippi, MD, U.O.

Diagnostica Ematochimica, Azienda

Ospedaliero-Universitaria di Parma,

Via Gramsci, 14, 43126 - Parma, Italy

(e-mail: glippi@ao.pr.it; ulippi@

tin.it).
Issue Theme Quality in Hemostasis and Thrombosis,

Part II; Guest Editors, Giuseppe

Lippi, MD, Mario Plebani, MD, and

Emmanuel J. Favaloro, PhD,

FFSC (RCPA).
Copyright $\odot 2013$ by Thieme Medical Publishers, Inc., 333 Seventh Avenue, New York, NY 10001, USA.

Tel: +1(212) 584-4662.
DOI http://dx.doi.org/

10.1055/s-0033-1334446. ISSN 0094-6176. 
"postanalytical" issues related to reporting of test results to the stakeholders-namely clinicians and patients) are the leading source of vulnerability in diagnostic testing, ${ }^{11}$ obviously also affecting the quality output of the hemostasis laboratory. ${ }^{12-14}$ Although due to dissimilar mechanisms and to differential extents, test results of clotting assays may be strongly influenced by the presence of common interfering substances, especially spurious (i.e., "in vitro") hemolysis, hyperbilirubinemia (i.e., icterus), and turbidity (i.e., lipemia). In the article by Lippi and colleagues, ${ }^{15}$ the impact on hemostasis testing of the presence of these substances is comprehensively discussed, including the leading mechanisms of interference, their impact on the specific clotting assays, as well as the recommended approaches to limit the likelihood of producing unsuitable test results and thus jeopardize patient safety. ${ }^{16}$ It is noteworthy that the type and degree of interference found in the different types of unsuitable specimens is remarkably different, so that distinctive approaches are specifically required to manage hemolyzed, icteric, and turbid samples.

The term lupus anticoagulants (LA) include a class of antiphospholipid antibodies that show marked interference with in vitro phospholipid-dependent clotting tests, although being paradoxically associated with a kaleidoscope of thrombotic manifestations, including recurrent venous and/or arterial thrombosis, as well as complications of pregnancy. ${ }^{17}$ The current diagnostic testing for LA is an essential part of diagnosis and management of patients with antiphospholipid syndrome (APS) and other hypercoagulable states, ${ }^{18}$ although there are several issues that make the pertinent investigations challenging and highly vulnerable to preanalytical, analytical, as well as postanalytical problems. ${ }^{19-22}$ The leading issues in APS testing include the heterogeneous sensitivity of tests and reagents, the clinically meaningful rate of falsenegative and false-positive results, an absence of consensus for the use of mixing tests, ${ }^{23,24}$ and a well-known lack of compliance with current guidelines. ${ }^{25}$ In the article by Adams, ${ }^{26}$ these issues are each reviewed according to the perspective of updated guidelines and according to results of recent external quality assessment (EQA) surveys.

In the next article, Duncan and colleagues ${ }^{27}$ provide a timely update about the well-known issue of factor VIII (FVIII) assessment in routine and specialized laboratories. ${ }^{28}$ Basically, several different methods are used for measuring the plasma concentration of FVIII; these entail one-stage or two-stage clotting assays, chromogenic tests, and immunoassays. The chromogenic tests are a form of two-stage assay and use activated factor X (FXa) enzymatic activity as a surrogate measure of FVIII cofactor activity on factor IX (FIX). The one-stage clotting assays, which are mostly based on a modification of the activated partial thromboplastin time (aPTT), represent the methodology used by most laboratories. Interestingly, one-stage clotting assays may provide FVIII levels that are up to $50 \%$ lower than those of the twostage clotting, chromogenic and immunological assays when used in patients with hemophilia A undergoing treatment with B-domain-deleted recombinant FVIII or full-length recombinant FVIII. ${ }^{28}$ It is noteworthy, however, that a subgroup of patients with mild hemophilia A (up to 40\%) display method-related biases in FVIII:C results, where the one-stage clotting assay instead provides significantly higher results than the two-stage clotting or the chromogenic assays. ${ }^{27}$ With the aim of identifying these patients, Duncan et al recommend that automated chromogenic assays should replace the challenging, manual two-stage clotting methods, and provide a comprehensive set of suggestions for obtaining accurate results, especially in the abnormal range of values. ${ }^{27}$ This may be critical to reliably reflect the clinical scenario and thereby prevent misdiagnosis or misclassification of this otherwise clinically significant bleeding disorder. The authors also endorsed that due to the limited array of FVIII gene (F8) missense mutations observed in these subset of mild hemophilia A patients, molecular analysis of the $A 1, A 2$, or $A 3$, domains of FVIII should be advisable for guiding diagnostic, clinical, and therapeutic decision making.

In the subsequent article, Kershaw and Orellana discuss a foremost topic for the coagulation laboratory, that is, the issue of mixing tests. ${ }^{29}$ Prolonged values of prothrombin time (PT), aPTT, or both, are a rather common occurrence, which may reflect clinically threatening or "benign" deficiencies of clotting factors, ${ }^{30}$ other bleeding disorders such as acquired inhibitors of coagulation factors (i.e., acquired hemophilia), ${ }^{31-33}$ the presence of LA, ${ }^{18-26}$ as well as other less frequent physiological or pathological conditions. ${ }^{34}$ Basically, a mixing test is based on the combination of an abnormal patient sample with "normal" pooled plasma (NPP), followed by duplication of the abnormal screening test on this mixture to establish as to whether the clotting time normalizes ("factor deficiency") or instead remains abnormal ("factor inhibitor"). Therefore, the inherent aim of this relatively simple and inexpensive process is to obtain suitable information about the potential source of prolongation of PT, aPTT, or both (- Fig. 1). As noted earlier, a normalization of the clotting time on mixing usually reflect an acquired or inherited clotting factor deficiency(s), whereas incomplete normalization is always suggestive for the presence of inhibitory substance(s). In the latter case, high dose heparin or antibodies (i.e., LA, specific coagulation factor inhibitors, or another type of inhibitor)-that ultimately interfere with the clotting process, by prolongation of the clotting time(s)-could be present, and this would need to be specifically evaluated by additional testing. In this article, the authors thereby discuss the leading objective triggering mixing test(s), the pertinent technical features (e.g., sensitivity of reagents, composition of the NPP, different formulas for expressing test results), as well as the recommended interpretative criteria and the subsequent diagnostic actions. ${ }^{29}$

The next article by Bunimov and colleagues outlines the genetic loci associated with platelet traits and platelet disorders. $^{35}$ Genetic investigations have led to important advances in our knowledge of genes, proteins, and micro ribonucleic acid that influence various aspects of circulating platelet counts, size, and function. The application of genome wide association studies (GWAS) to platelet traits has also identified multiple loci with significant association to platelet number, size, and function in aggregation and granule secretion assays. Moreover, the genes altered by disease causing 


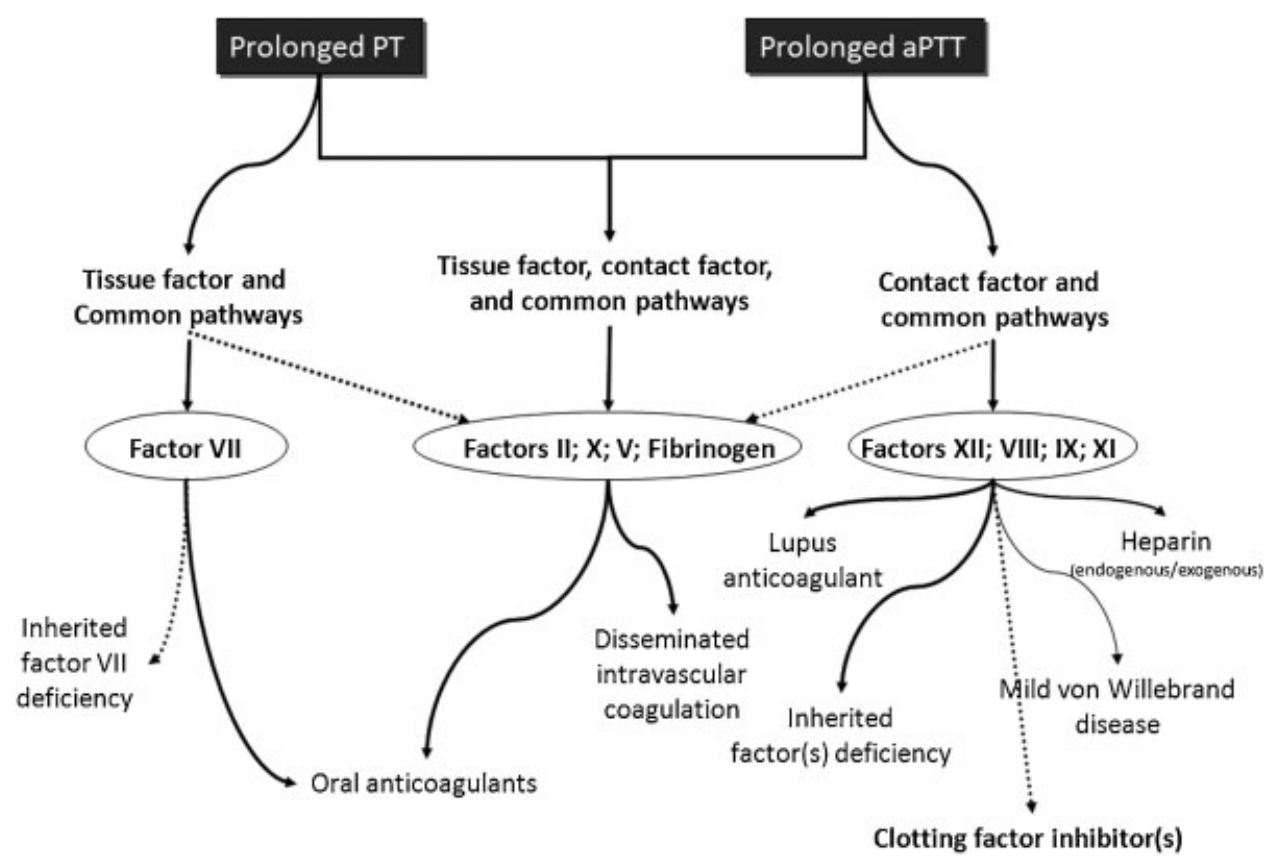

Fig. 1 Troubleshooting abnormal results of prothrombin time (PT) and activated partial thromboplastin time (aPTT). Tissue factor pathway = "extrinsic" in older terminology; contact factor pathway = "intrinsic" in older terminology.

mutations have now been identified for several platelet disorders, including X-linked recessive, autosomal dominant, and autosomal recessive platelet disorders. Nevertheless, the genetic mutations that cause common inherited platelet disorders, and impair platelet aggregation and granule secretion, remain largely unknown. This review summarizes current knowledge on the genetic loci that influence platelet traits, including the genes with well characterized mutations in certain inherited platelet disorders.

The last three articles in this issue comprise original articles. In the first of these, Fuchs and colleagues investigate the distinct role of von Willebrand factor (VWF) triplet bands in glycoprotein Ib-dependent platelet adhesion and thrombus formation under flow. ${ }^{36}$ These authors found that under highshear flow conditions, a VWF preparation enriched in intermediate triplet bands was less active in recruiting platelets to collagen type III than a VWF preparation with similar multimeric distribution but prevalently containing larger triplet bands. It was also observed that defective thrombus formation was less restored when the former preparation was tested with blood from patients with von Willebrand disease (VWD). It was therefore concluded that VWF forms that lack larger size triplet bands seem to display a lowered ability to recruit platelets to collagen-bound VWF under arterial flow conditions, so that variations in triplet band distribution observed in patients with VWD may be associated with abnormal platelet adhesiveness under high-shear flow conditions.

In the second of the original articles, McGlasson and Fritsma return us to the issue of screening and diagnostic testing in hemostasis, and namely assessment of LA. ${ }^{37}$ McGlasson and Fritsma assessed six dilute Russell viper venom time (DRVVT) LA screen and confirm assays to test intermethod consistency using a large number of normal and LA positive plasmas. Reagents were obtained from Diagnostica Stago Inc (Parsip- pany, NJ), Precision BioLogic Inc (Dartmouth, Nova Scotia, Canada), Siemens Healthcare (Malvern, PA), T Coag (Bray Co. Wicklow, Ireland), Instrumentation Laboratory (Bedford, MA), and Sekisui Diagnostics (Framingham, MA); and all were assayed an STA-R Evolution-automated coagulometer following manufacturer's instructions. It is noteworthy that DRVVT screen/confirm ratios for all but one manufacturer product were found able to discriminate between LA-positive and LA-negative samples, providing satisfactory imprecision (i.e., typically lower than 6\%) and acceptable agreement among the various assays, although a certain degree of variability was observed in the different reagent-instrument combinations. It was also interestingly found that normalization was virtually ineffective in improving the clinical interpretation of test results at the local level. Nevertheless, normalization of test results is still considered important in the context of harmonization of test results across methodologies and across laboratories, and analogous to the international normalized ratio test system for monitoring of vitamin $\mathrm{K}$ antagonist therapy.

The final article in this issue is by Bonar and colleagues. ${ }^{38}$ As noted earlier, inhibitors to coagulation factors cause prolongation of routine hemostasis laboratory test results, such as PT and aPTT. Inhibitors of FVIII have particular clinical relevance in the treatment and management of hemophilia patients, and can be either alloantibodies (as in hemophilia A), or autoantibodies (as in acquired hemophilia). ${ }^{31,33}$ The most commonly used assays for detecting these inhibitors are the classical Bethesda assay or a modified (Nijmegen) method. Previous laboratory assessments from EQA programs have shown a wide variability in FVIII inhibitor results and methods performed, as well as a significant degree of false positive and false negative interpretations. The Bethesda assay is still the primary assay used in laboratories for detecting the 
presence and strength of a FVIII inhibitor. Therefore, it is of utmost importance that this assay is appropriately performed. The current report reviews the most recent findings from the Royal College of Pathologists of Australasia Quality Assurance Program Haematology, which show there is still a need for better standardization and improvement in the detection of low-level FVIII inhibitors to ultimately provide better clinical management of affected patients in the future.

We wish, as usual, to congratulate and thank all authors to this issue of Seminars in Thrombosis E Hemostasis for their unique and comprehensive contributions, and we hope that our readership will find interest in the issue's contents.

\section{References}

1 Favaloro EJ, Plebani M, Lippi G. Quality in hemostasis and thrombosis-part I. Semin Thromb Hemost 2012;38(6):549-552

2 Plebani M, Lippi G. To err is human. To misdiagnose might be deadly. Clin Biochem 2010;43(1-2):1-3

3 Favaloro EJ, Plebani M, Lippi G. Regulation in hemostasis and thrombosis: part I-in vitro diagnostics. Semin Thromb Hemost 2013;39(3):235-249

4 Favaloro EJ. Standardization, regulation, quality assurance and emerging technologies in hemostasis: issues, controversies, benefits, and limitations. Semin Thromb Hemost 2007;33(3):290-297

5 Favaloro EJ, Plebani M, Lippi G. Regulation of in vitro diagnostics (IVDs) for use in clinical diagnostic laboratories: towards the light or dark in clinical laboratory testing? Clin Chem Lab Med 2011;49 (12):1965-1973

6 Favaloro EJ. Regulation of in vitro diagnostics (IVDs) for use in Australian pathology laboratories: a gloomy outlook for future pathology testing in this country? Pathology 2011;43(4):397-402

7 Favaloro EJ. Harmonising quality to the lowest clinical diagnostic standard? The case against regulation of in vitro diagnostics (IVDs) for use in clinical diagnostic laboratories. Aust J Med Sci 2010; 31:56-64

8 Lippi G, Franchini M, Targher G. Arterial thrombus formation in cardiovascular disease. Nat Rev Cardiol 2011;8(9):502-512

9 Lippi G, Franchini M. Pathogenesis of venous thromboembolism: when the cup runneth over. Semin Thromb Hemost 2008;34(8): 747-761

10 Simoens S, Huys I. Market entry of biosimilar low-molecularweight heparins in Europe: opportunities and challenges. Semin Thromb Hemost 2013;39(3):250-257

11 Plebani M, Lippi G. Closing the brain-to-brain loop in laboratory testing. Clin Chem Lab Med 2011;49(7):1131-1133

12 Favaloro EJ, Lippi G, Adcock DM. Preanalytical and postanalytical variables: the leading causes of diagnostic error in hemostasis? Semin Thromb Hemost 2008;34(7):612-634

13 Adcock Funk DM, Lippi G, Favaloro EJ. Quality standards for sample processing, transportation, and storage in hemostasis testing. Semin Thromb Hemost 2012;38(6):576-585

14 Lippi G, Salvagno GL, Montagnana M, Lima-Oliveira G, Guidi GC, Favaloro EJ. Quality standards for sample collection in coagulation testing. Semin Thromb Hemost 2012;38(6):565-575

15 Lippi G, Plebani M, Favaloro EJ. Interference in coagulation testing: focus on spurious hemolysis, icterus and lipemia. Semin Thromb Hemost 2013;39(3):258-266

16 Plebani M, Favaloro EJ, Lippi G. Patient safety and quality in laboratory and hemostasis testing: a renewed loop? Semin Thromb Hemost 2012;38(6):553-558

17 Pengo V, Banzato A, Bison E, Bracco A, Denas G, Ruffatti A. What have we learned about antiphospholipid syndrome from patients and antiphospholipid carrier cohorts? Semin Thromb Hemost 2012;38(4):322-327

18 Kershaw G, Suresh S, Orellana D, Nguy YM. Laboratory identification of lupus anticoagulants. Semin Thromb Hemost 2012;38 (4):375-384

19 Favaloro EJ. Trials and tribulations in lupus anticoagulant testing. Clin Chem Lab Med 2013;51(2):253-256

20 Pradella P, Azzarini G, Santarossa L, et al. Cooperation experience in a multicentre study to define the upper limits in a normal population for the diagnostic assessment of the functional lupus anticoagulant assays. Clin Chem Lab Med 2013;51(2):379-385

21 Froom P, Barak M. Testing for lupus anticoagulants-fresh or frozen? Clin Chem Lab Med 2012;50(9):1607-1609

22 Froom P, Barak M. Lupus anticoagulant testing: analyzing fresh samples after a single centrifugation and after a 6-8. h delay. Clin Chem Lab Med 2012;50(2):367-370

23 Tripodi A. Laboratory testing for lupus anticoagulants: diagnostic criteria and use of screening, mixing, and confirmatory studies. Semin Thromb Hemost 2008;34(4):373-379

24 Tripodi A. To mix or not to mix in lupus anticoagulant testing? That is the question. Semin Thromb Hemost 2012;38(4):385-389

25 Pengo V, Tripodi A, Reber G, et al; Subcommittee on Lupus Anticoagulant/Antiphospholipid Antibody of the Scientific and Standardisation Committee of the International Society on Thrombosis and Haemostasis. Update of the guidelines for lupus anticoagulant detection. J Thromb Haemost 2009;7(10):1737-1740

26 Adams M. Measurement of lupus anticoagulants: an update on quality in laboratory testing. Semin Thromb Hemost 2013; 39(3):267-271

27 Duncan EM, Rodgers SE, McRae SJ. Diagnostic testing for mild hemophilia A in patients with discrepant one-stage, two-stage and chromogenic factor VIII:C assays. Semin Thromb Hemost 2013; 39(3):272-282

28 Lippi G, Franchini M, Favaloro EJ. One-stage clotting versus chromogenic assays for assessing recombinant factor VIII: two faces of a haemostasis coin. Blood Coagul Fibrinolysis 2009;20(1):1-3

29 Kershaw G, Orellana D. Mixing tests: diagnostic aides in the investigation of prolonged prothrombin times and activated partial thromboplastin times. Semin Thromb Hemost 2013;39(3): 283-290

30 Lippi G, Franchini M, Montagnana M, Favaloro EJ. Inherited disorders of blood coagulation. Ann Med 2012;44(5):405-418

31 Coppola A, Favaloro EJ, Tufano A, Di Minno MN, Cerbone AM, Franchini M. Acquired inhibitors of coagulation factors: part Iacquired hemophilia A. Semin Thromb Hemost 2012;38(5): 433-446

32 Franchini M, Lippi G, Favaloro EJ. Acquired inhibitors of coagulation factors: part II. Semin Thromb Hemost 2012;38(5):447-453

33 Kershaw G, Favaloro EJ. Laboratory identification of factor inhibitors: an update. Pathology 2012;44(4):293-302

34 Lippi G, Favaloro EJ. Activated partial thromboplastin time: new tricks for an old dogma. Semin Thromb Hemost 2008;34(7): 604-611

35 Bunimov N, Fuller N, Hayward CPM. Genetic loci associated with platelet traits and platelet disorders. Semin Thromb Hemost 2013;39(3):291-305

36 Fuchs B, de Witt S, Solecka BA, et al. Distinct role of von Willebrand factor triplet bands in glycoprotein Ib-dependent platelet adhesion and thrombus formation under flow. Semin Thromb Hemost 2013;39(3):306-314

37 McGlasson DL, Fritsma GA. Comparison of six dilute Russell viper venom time lupus anticoagulant screen/confirm assay kits. Semin Thromb Hemost 2013;39(3):315-319

38 Bonar RA, Favaloro EJ, Marsden K. External quality assurance for factor VIII inhibitor assays. Semin Thromb Hemost 2013;39(3): $320-326$ 